AbstractID: 10625 Title: Comparing the Sensitivity of Ionization Chamber Array to Film and Point Dose Measurement for IMRT Delivery Errors

\title{
Purpose:
}

To compare the sensitivity of 2D ion chamber array, film dosimetry and point dose measurement for detecting IMRT delivery errors.

\section{Method and Materials:}

2 types of IMRT delivery errors were considered. First type involved adding $1 \mathrm{~mm}$ systematic error to 1 bank of the MLC leaves, resulting in a dose discrepancy of $\geq 5 \%$. The second type involved 'undeliverable intensity patterns', resulting from improper use of smoothness constraint during optimization. 4 and 2 plans were studied for type 1 and type 2 errors respectively.

$2 \mathrm{D}$ dose distribution was measured by 2D array (MatriXX) and film (EDR2). IBA CC04 chamber was used for point dose measurement. Measurements were done for composite plans with plan-specific gantry angles (2D array \& film + point dose) and gantry fixed at $0^{\circ}$ (2D array only). For type 2 error, we also measured dose profile of individual fields (2D array \& film). Error detection threshold was set at $90 \%$ for $3 \% / 3 \mathrm{~mm}$ criteria of the $\gamma$ analysis.

\section{Results:}

For $1 \mathrm{~mm}$ systematic error in MLC positions, when the plans were delivered with the planspecific gantry angles, both array and film were able to reject all the faulty plans. If the plans were delivered at $0^{\circ}$, the $2 \mathrm{D}$ array detected only 1 out of the 4 error plans. For the 'undeliverable intensity patterns', the 2D array, film and absolute point dose measurement were able to reject both plans when composite plans were delivered at plan-specific beam angles. Irradiating composite plans with gantry fixed at $0^{\circ}$ failed to spot errors in them, although errors were found when analyzing individual fields.

\section{Conclusions:}

Our study showed limitation of 2D array in detecting delivery errors when using the single gantry angle composite plan approach. QA with plan specific gantry angles may be more robust in detecting errors in IMRT delivery. 